\title{
Adult Solid Neoplasm
}

National Cancer Institute

\section{Source}

National Cancer Institute. Adult Solid Neoplasm. NCI Thesaurus. Code C8101.

A solid neoplasm (e.g., carcinoma, sarcoma) occurring in adults. 